\title{
A Class of Marshall Type Maximal Inequalities for Conditional Demimartingales
}

\author{
Yali Lu, Decheng Feng, Xia Lin
}

\begin{abstract}
In this paper, we got a further studying of Marshall type inequalities. We obtained a class of Marshall type maximal inequalities for conditional demimartingales $\left\{S_{n}\right.$, n>1\} by using conditional Hölder inequalities and some inequalities related to conditional demimartingales.
\end{abstract}

Keywords-Conditional Demimartingales; Marshall Type Inequalities; Maximal Inequalities

\section{INTRODUCTION}

In this paper, let $\left\{S_{n}, n>1\right\}$ be a random variables sequence defined on $(\Omega, F, P)$. Denote $S_{0}=0, I(A)$ is indicator function of set $\mathrm{A}, \mathrm{p}>0, \mathrm{p} \neq 1$ and $\frac{1}{\mathrm{p}}+\frac{1}{q}=1$. Let $\Lambda=\left\{\max _{1 \leq \mathrm{k} \leq \mathrm{n}} \mathrm{S}_{\mathrm{k}} \geq \varepsilon\right\}$.

Definition 1 Let $\left\{S_{n}, n>1\right\}$ is a random variables sequence on $\mathrm{L}^{1}(\Omega, \mathrm{F}, \mathrm{P})$. If for all $1 \leq i<j<\infty$, $\mathrm{E}\left[\left(\mathrm{S}_{\mathrm{j}}-\mathrm{S}_{\mathrm{i}}\right) \mathrm{f}\left(\mathrm{S}_{1}, \ldots, \mathrm{S}_{\mathrm{j}}\right)\right]>0$

for every componentwise nondecreasing function $f$ where expectation is defined, then the sequence $\left\{S_{n}\right.$, $n>1\}$ is called a demimartingale. In addition, if assume $\mathrm{f}$ is nonnegative, then $\left\{\mathrm{S}_{\mathrm{n}}, \mathrm{n}>1\right\}$ is called a demisubmartingale.

The concept of demimartingale was introduced by Newman et al[1], after that many scholars got further studying about it and investigated some interesting results and applications[2-11].

For zero mean square integrable random variable $\mathrm{X}$, we have

$$
P\{X \geq \varepsilon\} \leq \frac{E X^{2}}{\varepsilon^{2}+E X^{2}}, \forall \varepsilon>0 .
$$

Marshall[2] generalized above inequality as following form

Yali Lu, School of Mathematics and Statistics, Northwest Normal University, Lanzhou, Gansu, China, Mobile No. 15002511178.
$P\left\{\max _{1 \leq k \leq n}\left(X_{1}+X_{2}+\cdots+X_{k}\right)\right\} \leq \frac{\sum_{k=1}^{n} E X^{2}}{\varepsilon^{2}+\sum_{k=1}^{n} E X^{2}}, \forall \varepsilon>0$

where $\mathrm{EX}_{\mathrm{k}}=0, \mathrm{E}\left(\mathrm{X}_{\mathrm{k}} \mid \mathrm{X}_{1}, \mathrm{X}_{2}, \ldots, \mathrm{X}_{\mathrm{k}-1}\right)=0$ a.e., $\mathrm{k}>$ 2 and $E X_{\mathrm{k}}^{2}<\infty, k>1$.

Under above condition, if assume

$$
S_{\mathrm{n}}=\sum_{k=1}^{n} X_{k}
$$

then $\left\{S_{n}, n>1\right\}$ would be a demimartingale. Mu et al[4] generalized inequality (1) under the condition of $E\left|X_{i}\right|^{p}<\infty, \mathrm{i}>1$ and $\mathrm{p}>2$, and got Marshall type inequality as following form

$P\left\{\max _{1 \leq k \leq n} S_{k} \geq \varepsilon\right\} \leq \frac{E\left|S_{n}\right|^{p}}{\alpha^{1-p} \varepsilon^{p}+E\left|S_{n}\right|^{p}}, \forall \varepsilon>0$

where $\alpha$ is maximum value of following function $h(x)=1-x+(1-x)^{2-q} x^{q-1}, x \in[0,1]$.

After that, $\mathrm{Hu}$ et al[5] generalized results in literature [4] to the case of condition demimartingales and obtained the Marshall type probability inequalities for demimartingales.

Assume $\mathrm{X}$ and $\mathrm{Y}$ are random variables defined on probability space $(\Omega, \mathrm{F}, \mathrm{P})$ and $E X^{2}<\infty, E Y^{2}<\infty$. Let $\mathrm{F}$ be a sub $\sigma$-algebra of $\mathrm{A}$,

Prakasa Rao[14] defined conditional covariance of $\mathrm{X}$ and $\mathrm{Y}(\mathrm{F}$-covariance $)$ as follow

$\operatorname{Cov}^{F}=E^{F}\left(\left(X-E^{F} X\right)\left(Y-E^{F} Y\right)\right)$

where $\mathrm{E}^{F} \mathrm{Z}$ is conditional expectation of random variable $\mathrm{Z}$ which has been given condition $F$.

Christofides et al[12] introduced following definition.

Definition 2 Let $\left\{S_{n}, n>1\right\}$ be a random variables 
sequence on $\mathrm{L}^{1}(\Omega, \mathrm{F}, \mathrm{P})$, if for all $1 \leq i<j<\infty$,

$E^{F}\left[\left(S_{j}-S_{i}\right) f\left(S_{1}, \cdots S_{j}\right)\right] \geq 0$ a.s.

for every componentwise nondecreasing function $f$ such that above expectation is defined, then $\left\{S_{n}, n>\right.$ $1\}$ is called a F-demimartingale. In addition, if assume $\mathrm{f}$ is nonnegative, then $\left\{\mathrm{S}_{\mathrm{n}}, \mathrm{n}>1\right\}$ is called a $\mathrm{F}$ demisubmartingale.

Inspired by literature[13], we extend Marshall type maximal inequalities for nonnegative demimartingales $\left\{S_{n}, n>1\right\}$ in literature [4] to the case of conditional demimartingales $\left\{S_{n}, n>1\right\}$.

\section{MAXIMAL INEQUALITIES FOR CONDITIONAL DEMIMARTINGALES}

Lemma 3[15] If $E^{F}|X|^{\mathrm{p}}<\infty$ a.s., $E^{F}|Y|^{\mathrm{p}}<\infty$ a.s., then

$E^{F}|X Y| \leq\left(E^{F}|X|^{p}\right)^{\frac{1}{p}}\left(E^{F}|Y|^{q}\right)^{\frac{1}{q}}$ a.s. , p >1,

$$
E^{F}|X Y| \geq\left(E^{F}|X|^{p}\right)^{\frac{1}{p}}\left(E^{F}|Y|^{q}\right)^{\frac{1}{q}} \text { a.s. }, 0<\mathrm{p}<1 .
$$

Lemma $4[12]$ Let $\left\{S_{n}, n>1\right\}$ be a F-demi(sub)martingale and $\mathrm{g}(\cdot)$ be a nonnegative nondecreasing convex function, then $\left\{\mathrm{g}\left(\mathrm{S}_{\mathrm{n}}\right), \mathrm{n}>1\right\}$ is a F-demisubmartingale.

Lemma 5[13] Let $\left\{S_{n}, n>1\right\}$ be a F-demisubmartingale, then for any F-measurable random variable $\varepsilon>0$ a.s.

$\varepsilon P^{F}\left(\max _{1 \leq k \leq n} S_{k} \geq \varepsilon\right) \leq E^{F}\left(S_{n} I\left(\max _{1 \leq k \leq n} S_{k} \geq \varepsilon\right)\right)$ a.s. (4)

Lemma 6 Let $\left\{S_{n}, n>1\right\}$ be a F-demisubmartingle and $\mathrm{E}^{F} \mathrm{~S}_{1} \leq 0$ a.s., assume there is a $1<\mathrm{p}<2$, such that $E^{F}\left|S_{n}\right|^{p}<\infty$ a.s. for all $\mathrm{n}>1$ established, then for every F-measurable random variable $\varepsilon>0$ a.s. while $P(\Lambda) \in\left[\frac{1}{2}, 1\right]$ a.s.

$$
\begin{aligned}
& {\left[P^{F}(\Lambda)\left(1-P^{F}(\Lambda)\right)^{\mathrm{q}}+\left(1-P^{F}(\Lambda)\right) P^{F}(\Lambda)^{q}\right]^{\frac{1}{q}}} \\
& \left(E^{F}\left|S_{n}\right|^{p}\right)^{\frac{1}{p}} \geq \varepsilon P^{F}(\Lambda)
\end{aligned}
$$$$
\text { a.s }
$$

Proof. Denote $\mathrm{Y}=\mathrm{I}(\Lambda)$. By using conditional Hölder inequality (2) and lemma 5, we can get

$$
\left(E^{F}\left|Y-E^{F} Y\right|^{q}\right)^{\frac{1}{q}}\left(E^{F}\left|S_{n}\right|^{p}\right)^{\frac{1}{p}}
$$

$\geq E^{F}\left[\left(Y-E^{F} Y\right) S_{\mathrm{n}}\right]$

$=E^{F}\left(Y S_{n}\right)-E^{F} Y E^{F} S_{n}$

$\geq E^{F}\left(I(\Lambda) S_{n}\right)$

$\geq E^{F}(\varepsilon I(\Lambda))$

$=\varepsilon P^{F}(\Lambda)$

a. s.

since

$P^{F}(\Lambda) \leq P^{F}(\Lambda)\left(1-P^{F}(\Lambda)\right)^{\mathrm{q}}+\left(1-P^{F}(\Lambda)\right) P^{F}(\Lambda)^{q}$ a.s.

where $P^{F}(\Lambda) \in\left[\frac{1}{2}, 1\right]$ a.s., thus the proposition is proved.

Theorem 7 Let $\left(\mathrm{Sn}, \mathrm{n}>1\right.$ \} be a F-demimartingale, $\mathrm{E}^{F} \mathrm{~S}_{1}<$ 0 . If there is a $1<\mathrm{p}<2$, such that for all $\mathrm{n}>1$, have $0<E^{F}\left|S_{\mathrm{n}}\right|^{p}<\infty$ a.s., then for every F-measurable random variable $\varepsilon>0$ a.s. while $P^{F}(\Lambda) \in\left[\frac{1}{2}, 1\right]$ a.s.,we have

$P^{F}(\Lambda) \leq \frac{1}{1+M}$ a.s.

where $\mathrm{M}$ is positive solution of following function,

$x^{q}=(\beta-1) x+\beta, x \in(0,+\infty)$.

where $\beta=\frac{\varepsilon^{q}}{\left(E^{F}\left|S_{n}\right|^{p}\right)^{\frac{q}{p}}}$ a.s.

Proof. By lemma 6 we can get

$\left[P^{F}(\Lambda)\left(1-P^{F}(\Lambda)\right)^{q}+\left(1-P^{F}(\Lambda)\right) P^{F}(\Lambda)^{q}\right] \geq \varepsilon^{q} P^{F}(\Lambda)^{q}$ a.s.

divide both side by $\mathrm{P}^{\mathrm{F}}(\Lambda)^{\mathrm{q}}$

$\left[P^{F}(\Lambda) \frac{\left(1-P^{F}(\Lambda)\right)^{q}}{P^{F}(\Lambda)^{q}}+\left(1+P^{F}(\Lambda)\right)\right]\left(E^{F}\left|S_{n}\right|^{p}\right)^{\frac{q}{p}} \geq \varepsilon^{q}$ a.s.

let $x_{0}=\frac{1-P^{F}(\Lambda)}{P^{F}(\Lambda)}$ a.s., $\beta=\frac{\varepsilon^{q}}{\left(E^{F}\left|S_{n}\right|^{p}\right)^{\frac{q}{p}}}$ a.s., then

$P^{F}(\Lambda)=\frac{1}{1+x_{0}}$ a.s.

since 


$$
P^{F}(\Lambda) x_{0}{ }^{q}+1-P^{F}(\Lambda) \geq \beta \text { a.s. }
$$

then

$\frac{x_{0}{ }^{q}}{1+x_{0}}+\frac{x_{0}}{1+x_{0}} \geq \beta$ a.s.

namely

$x_{0}^{q} \geq(\beta-1) x_{0}+\beta$ a.s.

(6)

let $g(x)=x^{q}-(\beta-1) x_{0}+\beta, \mathrm{M}$ is positive solution of equation(5). Since $\quad g^{\prime \prime}(x)=q(q-1) x^{q-2}>0$ $x \in(0,+\infty)$, we can easily get $\mathrm{g}(\mathrm{x})$ is a convex function on interval $(0,+\infty)$, which mean for $\forall x \in(0, M)$

$\frac{g(x)-g(0)}{x-0} \leq \frac{g(M)-g(x)}{M-x}$,

since $g(0)=-\beta<0$ and $g(M)=0$. Then for $x \in(0, M)$ can get $\mathrm{g}(x)<0$, namely $\mathrm{M}$ is minimum value of equation (6).

This work was supported by the National Natural Science Foundation of China(Grant No. 11861057 and No. 11761064), Innovation Ability Promotion Project of Colleges and Universities in Gansu Province(Grant No. 2019A-003), Research Funding Project for Postgraduates in Northwest Normal University(Grant No. 2020KYZZ001113).

\section{REFERENCES}

[1] NEWMAN C M, WRIGHT A L. Associated random variables and martingale inequalities[J].Zeitschrift Für Wahrscheinlichkeitstheorie and Verwandte Gebiete, 1982, 59(3): 361-371.

[2] MARSHALL A W. A one-sided analog ofKolmogorovs inequality[J]. Ann MathematicalStatistics, 1960, 31(2): 483-487.

[3] HU S H, WANG X J, YANG W Z. The Hàjek-Rènyi-type inequality for associatedrandom variables $[\mathrm{J}]$. Statistics and ProbabilityLetters, 2009, 79(7): 884-888.

[4] MU J Y, MIAO Y.Generalizing the Marshall's inequality $[\mathrm{J}]$. Communications in Statistics Theoryand Methods,2011, 40(15): 2809-2817.

[5] HU S H, WANG X J, YANG W Z. Someinequalities for demimartingales andN-martingales[J]. Statistics and
ProbabilityLetters, 2012, 82(2): 232-239.

[6] CHRISTOFIDES T C. U-statistics on

associatedrandom variables[J]. J Statistical Planning andInference, 2004, 119(1): 115

[7] Dai Ping-ping, SHEN Yan, HU Shu-he. SomeResults for demimartingales andN-demimartingales[J]. Journal of Inequalities andApplications, 2014(1): 489-501.

[8] PRAKASA RAO B L S. On some maximal inequalities for demisubmartingales and $\mathrm{N}$-demisubmartingales[J]. Journal of Inequalities in Pure and Applied Mathematics, 2007, 8(4): 112.

[9] PRAKASA RAO B L S. Remarks on maximalinequalities for nonnegative demisubmartingales[J].Statistics and Probability Letters, 2012, 82(7):1388-1390.

[10] CHRISTOFIDES T C. Maximal inequalitiesfor Ndemimartingales[J]. Arch Inequal Appl,2003, 50:397-408.

[11] WANG J F. Maximal inequalities for associatedrandom variables and demimartingales[J]. Statistics and Probability Letters, 2004, 66(3): 347-354.

[12] CHRISTOFIDES T C, Hadjikyriakou M.Conditional demimartingales and related results.Journal of Mathematical Analysis andApplications, 2013, 298(1): 308-391

[13] WANG X H, WANG X J. Some inequalities for conditional demisubmartingales andconditional N-demisubmartingales[J]. Statisticsand Probability Letters, 2013, 83(7): 700-709.

[14] PRAKASA RAO B L S. Conditional independence, conditional mixing and conditionalassociation $[\mathrm{J}]$. Annals of the Institute ofStatitical Mathematics, 2009, 61(2): 441-460.

[15] Chow Y. S., Teicher H. Probability Theory: Independence, Interchangebility, Martingales, second ed Spring-Verlag, New York, 1988.

Yali Lu, School of Mathematics and Statistics, Northwest Normal University, Lanzhou, China, Mobile 86-15002511178

Decheng Feng, School of Mathematics and Statistics, Northwest Normal University, Lanzhou, China.

Xia Lin, School of Mathematics and Statistics, Northwest Normal University, Lanzhou, China. 